\section{Automonitoração da Glicemia no Diabetes Mellitus do Tipo 1: Um Investimento com Retorno Garantido}

$\mathrm{O}$ diabetes mellitus (DM) está se tornando um dos problemas mais significativos de saúde pública não apenas nos países desenvolvidos mas também naqueles em desenvolvimento. No momento, estima-se que tenhamos mais de 140 milhões de indivíduos com DM no mundo e as projeções indicam que teremos mais de 230 milhões de pessoas afetadas por volta do ano 2010 . Aproximadamente $10 \%$ desta população é portadora de diabetes mellitus do tipo 1 (DMI).

Estudos recentes mostraram que tanto para o DMl (Diabetes Control and Complications Trial -DCCT) (1) como para o DM2 (United Kingdon Prospective Diabetes Study - UKPDS) (2) o controle metabólico dentro de determinados limites (níveis de glicohemoglobina inferior a $1,2 \%$ do limite superior da normalidade para o método no DMl e inferior a $1 \%$ no DM2) é capaz de diminuir significativamente o desenvolvimento das complicações microangiopáticas.

Nesse sentido, analisando a média do nível de controle da grande maioria dos indivíduos diabéticos, esta necessita ser melhorada. Como exemplo, sabe-se que a média nacional do nível de glicohemoglobina entre os indivíduos com DM nos Estados Unidos da América (3) está entre 9 e $10 \%$ (aproximadamente 3 a $4 \%$ acima do limite normal superior). Nesse mesmo país, quase 20.000 pessoas tornam-se cegas anualmente em decorrência do DM. Quase 50\% dos pacientes que iniciam o tratamento dialítico o fazem devido à nefropatia diabética. Do mesmo modo, o custo pessoal e financeiro das amputações dos membros inferiores é assombroso. Isto, considerando um dos países do primeiro mundo; e o mesmo deve ser verdadeiro para o nosso país, talvez até em uma escala maior.

Uma mudança nessa situação atual compreenderia alterações que resultem em uma melhora a nível populacional do controle metabólico dos indivíduos com DM. O nível de controle dos pacientes com DM pode ser dividido em cincos estádios. $\mathrm{O}^{\circ}$ consiste em diminuir o número de internações hospitalares dos pacientes por hiperglicemia; o $2^{\circ}$, eliminar os sintomas e sinais da hiperglicemia, como poliuria, polidipsia e emagrecimento; o $3^{\circ}$, manter níveis glicêmicos satisfatórios; o $4^{\circ}$, atingir valores de glicohemoglobina adequados e o $5^{\circ}$, prevenir as complicações crônicas da doença e manter uma qualidade de vida boa e produtiva.

Neste número dos "Arquivos", Lemos-Marini e colaboradores (4) mostram o impacto da introdução da automonitoração domiciliar em uma clientela de pacientes com DMI de um Hospital Universitário, no número de internações por hiperglicemia. Isto é, no primeiro estágio do controle metabólico do diabetes. Esses autores mostram que apesar de um aumento na população de pacientes acompanhados, houve uma redução no número de internações e esta mostrou uma correlação negativa com o número de tiras para glicosúria fornecidas. $\mathrm{O}$ mesmo não foi encontrado para o número de tiras para glicemia.

Estes achados sugerem os seguintes comentários adicionais:

1) $\mathrm{O}$ fato da distribuição das tiras reagentes coincidir com o início de funcionamento do grupo multidisciplinar na atenção a esses pacientes pode ter colaborado para o resultado encontrado, desde que estudos anteriores editorial

\author{
Sergio Atala Dib
}


$(5,6)$ já demonstraram uma redução de 25 a $40 \%$ no número de hospitalizações em pacientes que recebem uma educação sistemática sobre o diabetes.

2) Alguns aspectos devem ser considerados quando utilizamos a glicosúria para estimar a glicemia: a) a capacidade máxima de reabsorção tubular renal de glicose (Tm de glicose) corresponde a uma concentração plasmática de glicose de aproximadamente $10 \mathrm{mM}$ ou $180 \mathrm{mg} / \mathrm{dl}$. De modo que para glicemias em torno de $180 \mathrm{mg} / \mathrm{dl}$ a glicosúria deve ser negativa. Crianças podem ter um Tm de glicose muito baixo ou variável, resultando em glicosúria com euglicemia; b) a ingesta de líquidos e a concentração urinária podem alterar os testes; c) uma glicosúria negativa não é capaz de fazer distinção entre hipoglicemia, euglicemia ou mesmo uma hiperglicemia leve ou moderada; d) a metodologia para os testes de urina domiciliares envolvem o uso de tiras reagentes que mudam de cor e são comparadas com um padrão. Isto torna-se difícil para indivíduos daltônicos e para pacientes com comprometimento visual; e) algumas drogas (como p.ex.: vitamina $\mathrm{C}$ e aspirina) podem falsear as determinações da glicosúria.

As considerações acima devem ser de conhecimento da equipe multidisciplinar e dos pacientes, para que possam interpretar adequadamente as glicosúrias e saber as suas limitações. Entretanto, a monitoração da glicosúria ainda não é um método totalmente obsoleto já que é barata, indolor e pode ser utilizada pela grande maioria de pacientes, como a deste estudo (4), até que métodos mais econômicos e/ou não invasivos de avaliação da glicemia estejam disponíveis.

3) Atualmente os métodos de escolha para automonitoração do tratamento da hiperglicemia dos pacientes diabéticos são a glicemia capilar e a glicohemoglobina. Já existem aparelhos comercializados que determinam estes dois parâmetros a nível domiciliar. Entretanto, os mais utilizados são os glicosímetros. Os programas de autocontrole domiciliar da glicemia capilar são muito variáveis. Alguns esquemas recomendam quatro ou mais testes ao dia e outros mais simples, uma avaliação diária, mas em horários diferentes, de modo que todos os períodos e diferentes situações sejam avaliadas, por exemplo, no curso de uma semana. Um dos esquemas mais utilizado é o de quatro testes ao dia durante dois ou três dias na semana. Os pacientes mais motivados em melhorar significativamente o seu nível de controle metabólico monitoram a glicemia várias vezes ao dia $\mathrm{e}$ regularmente. Para a maioria destes pacientes, solicita-se verificar diariamente a glicemia antes de cada refeição e à noite ao deitar, além de uma vez por semana, também fazer o teste entre 2 e 3 horas da madrugada, com o objetivo de detectar hipoglicemias noturnas e, periodicamente, 2 a 3 horas após as refeições, para avaliar as excursões pós-prandiais da glicemia. Considera-se como bom controle glicêmico quando 80 a $90 \%$ das avaliações mostram glicemias de jejum entre 80 e $120 \mathrm{mg} / \mathrm{dl}, 1$ ? a 2 horas pós-prandiais entre 80 e $160 \mathrm{mg} / \mathrm{dl}$, e entre 2 e 3 horas da madrugada, superiores a $60 \mathrm{mg} / \mathrm{dl}$.

4) O número de visitas à clínica multidisciplinar de diabetes também tem uma correlação significativa com os níveis de $\mathrm{HbAlc}$, como demonstrado recentemente (7) em um estudo no qual os níveis desse parâmetro eram inferiores naqueles pacientes com maior aderência a um esquema de visitas quadrimensal durante um período de 3 anos.

Em 1993, membros da ISPAD (International Society for Pediatric and Adolescent Diabetes) elaboraram a denominada declaração de Kos para promover melhora do bem estar e qualidade de vida para todas as crianças e adolescentes com diabetes por volta do ano 2000 (8). Nesta declaração, entre os pontos principais estão a suplementação contínua de insulina, o acesso a uma equipe multidisciplinar de assistência e um aumento na disponibilidade de equipamentos e suprimentos para avaliação da glicosúria e glicemia para as crianças e adolescentes com DM.

O presente estudo (4) mostra que mesmo os passos iniciais para se atingir esses objetivos nos serviços públicos, abrangendo um grande número de DMl, já apresentam reflexos imediatos nas internações hospitalares com uma relação custo-beneficio positiva garantida.

\section{REFERÊNCIAS}

1. The Diabetes Control and Complications Trial Research Group: The effect of intensive treatment of diabetes on the development and progression of long-term complications in insulin-dependent diabetes mellitus. N Engl J Med 1996;329:977-86.

2. UK Prospective Diabetes Study Group: Effect of intensive blood-glucose control with sulphonylureas or insulin compared with conventional treatment and risk of complications in patients with type 2 diabetes. (UKPDS 33). Lancet 1998:352:837-53.

3. Davidson MB. Quality and the Art of Diabetes Maintenance. Professional Section Quartely, News From The American Diabetes Association 1997, Summer.

4. Lemos-Marini SHV, Lima MC, Guerra G Jr, Paulino MFVM, Minicucci WJ. A importância dos controles domiciliares na reducão de internações em portadores de diabetes mellitus do tipo 1. Arq Bras Endocrinol Metab 2000;44(3):215-9.

5. Scott RS, Brown LJ, Clifford P. Use of health services by diabetic persons. II Hospital admissions. Diabetes Care 1985:8:43-7.

6. Zaremba MM, Willhoite B, Ra K. Self-reported data: reliability and role in determining program effectiveness. Diabetes Care 1985;8:486-90.

7. Kaufman RF, Halvorson MSN, Carpenter S. Association between diabetes control and visits to a multidisciplinary pediatric diabetes clinic. Pediatrics 1999; 103:948-51.

8. Weber B, Brink S, Bartsocas C, Staehr-Johansen KJ. International Society for Pediatric and Adolescent Diabetes - ISPAD. Declaration of Kos (Letter). J Paediatr Child Health 1995:31:156. 